- 研究报告・

\title{
西藏湖泊长刺溞复合种的系统进化关系
}

\author{
谢理丽 徐 䂞 ${ }^{*}$ 林秋奇 韩博平
}

(暨南大学生态学系, 广州 510632)

\begin{abstract}
摘要: 本文通过对西藏湖泊长刺溞复合种(Daphnia longispina complex)中分布最广的 3 个物种, 即长刺溞(D. longispina)、灰形溞(D. galeata) 和颈齿溞( D. dentifera)线粒体COI基因序列以及GenBank中欧洲的长刺溞、加拿大的颈 齿溞和我国东部低海拔地区的灰型溞COI基因序列的比较分析, 研究了西藏湖泊长刺溞复合种的系统进化关系, 发现西藏地区的盔型溞、颈齿溞和长刺溞均已出现较大分化。颈齿溞种群内遗传差异度为 $0.33-2.32 \%$, 盔型溞为 0.33-2.74\%, 长刺溞的遗传差异度最高, 为1.31-5.50\%。基于COI基因序列构建的最大似然树和贝叶斯系统树均表 明, 长刺溞复合种由 3 个进化分支组成, 分别对应长刺溞、盔型溞和颈齿溞, 三者之间的遗传差异度为 9.40-16.98\%(Kimura 2-parameter双参数模型)。基于COI基因单倍型(haplotype)所构建的网络关系也支持上述3个分 支的存在。早期记录虽然显示长刺溞在我国分布较广, 但本次调查只在班公错有发现, 相比之下, 盔形溞和颈齿溞 则分布更广。我们的研究表明, 由于形态学鉴定上的局限性, 早期的长刺溞记录很可能混杂了容易引起混淆的盔 型溞或颈齿溞。
\end{abstract}

关键词: 溞属, 线粒体基因, 西藏, 系统进化

\section{Phylogenetics of the Daphnia longispina complex in Tibetan lakes}

Lili Xie, Lei Xu*, Qiuqi Lin, Boping Han

Department of Ecology, Jinan University, Guangzhou 510632

\begin{abstract}
To understand the phylogeography of the Daphnia longispina complex (consisting of three species: Daphnia longispina, Daphnia galeata and Daphnia dentifera) in the lakes of Tibet, we amplified the mitochondria COI sequences of the Daphnia longispina complex from Tibetan lakes and compared these with sequences from GenBank (containing Daphnia longispina from Europe, Daphnia galeata from the low altitudes area of eastern China, and Daphnia dentifera from Canada). Results showed that there is significant differentiation within Daphnia longispina, Daphnia galeata and Daphnia dentifera in the lakes of Tibet. The genetic diversity within Daphnia dentifera is $0.33-2.32 \%, 0.33-2.74 \%$ for Daphnia galeata, and 1.33-5.50\% for Daphnia longispina, representing the largest among the three species. Both Maximum Likelihood and Bayes trees based on mitochondria COI sequences showed that the Daphnia longispina complex was composed of three obvious clades, corresponding to Daphnia longispina, Daphnia galeata and Daphnia dentifera, respectively. The genetic diversity among the clades was $9.40-16.98 \%$, according to a Kimura 2-parameter model. Haplotype network based on the mitochondria COI sequences showed that the Daphnia longispina complex was composed of three branches, corresponding to Daphnia longispina, Daphnia galeata and Daphnia dentifera, respectively. Early Chinese records showed that Daphnia longispina was widely distributed, but in this present study, Daphnia longispina only appeared in Lake Bangongcuo, and Daphnia galeata and Daphnia dentifera were more widely distributed. Because of the difficulty in morphological identification as well as the lack of molecular data in early investigations, the early records of Daphnia longispina in China were probably confused with Daphnia galeata or Daphnia dentifera.
\end{abstract}

Key words: Daphnia, mitochondrial gene, Tibet, phylogeny

收稿日期: 2015-03-25; 接受日期: 2015-09-01

基金项目: 科技部 973 项目(2012CB956100)和国家自然科学基金(21314032)

* 通迅作者 Author for correspondence. E-mail: cloud_xu@hotmail.com 
枝角类是浮游动物的重要类群, 主要指节肢动 物门甲壳超纲瓣鳃纲枝角超目和薄皮溞超目中的 动物, 包括栉足目、异足目、钩足目和单足目 (Dumont \& Negrea, 2002)。目前全球共报道95属620 种(Forro' et al., 2008), 但这一数字被认为远远低估 了枝角类的物种多样性。Korovchinsky(1996)认为只 有 $45-50 \%$ 的枝角类种类得到完整的描述，而其他 种类描述不够明确, 并且大部分种类存在隐种 (cryptic species)。

溞属(Daphnia) 是枝角类中研究最为广泛的浮 游动物, 由于其超强的滤食能力以及在淡水生态系 统中的特殊地位 (Hutchison, 1967; De Meester, 1996), 溞属中的许多种类已被广泛作为模式生物, 用于生物毒理学、遗传学、生态学和进化生物学的 研究(Hebert, 1978; Lampert, 2011)。然而, 溞属的形 态分类一直很困难，对于溞属内不同种类的分类问 题已经争论了近一个世纪(Benzie, 2005; Kotov, 2015), 其分类困难的主要原因有以下几点: (1)个体 外形变化大, 特别是受环境影响导致(例如头盔的 形状变化与鱼类捕食压力有关); (2)用于形态鉴定 的特征分辨率不足; (3)种间生殖隔离不完全, 不同 种类可以通过杂交形成后代; (4)分布极广泛, 仅在 一个地区发现的模式标本很难完全代表其分类学 特征(Adamowicz \& Purvis, 2005)。随着分子生物学 与形态学方法的结合, 溞属中大量隐种被发现, 如 D. lacustris、D. cristata、D. hyalo等(Petrusek et al., 2007; Petrusek, 2007); 而近缘种间普遍存在的杂交 现象使得分类更加复杂与困难。溞属中许多种类以 复合种(complex species)的形式存在(Benzie, 1988; Kotov et al., 2006), 如长刺溞复合种(Daphnia longispina complex)、蚤状溞复合种(D. pulex complex)、 隆腺溞复合种(D. carinata complex)、同形溞复合种 (D. similis complex)、光滑溞复合种(D. laevis complex)等(Giessler, 1997a; Mergeay et al., 2008; Dlouhá et al., 2010)。在这些复合种中, 长刺溞复合种分布 最广, 种类也最多, 其复合种包括长刺溞( D. longispina)、僧帽溞(D. cucullata)、透明溞(D. hyalina)、 盔型溞 $(D$. galeata)、颈齿溞 $(D$. dentifera $) 、 D$. mendotae、D. rosea、D. thorala、D. umbra等 (Colbourne et al., 1998; Petrusek et al., 2008)。

目前, 欧洲和北美对长刺溞复合种的种群分化 与系统地理学的大量研究表明, 长刺溞复合种的种
群分化与地理距离没有明显的相关性，相邻栖息地 的种群遗传结构呈现明显的分化(Giessler, 1997b; Skage et al., 2007; Ventura et al., 2014)。Ishida等 (2007，2011)利用线粒体与核基因研究长刺溞复合 种内的几个种类(盔型溞和颈齿溞)的分子系统学, 发现新北区的盔型溞和颈齿溞在第四纪冰川活动 中存在基因渗透现象, 而在日本岛分布的盔型溞和 颈齿溞受鱼类捕食压力和气候变化影响, 其分布呈 现区域分化, 盔型溞主要分布于低纬度或低海拔地 区, 而颈齿溞主要分布于高纬度或高海拔地区 (Allen et al., 2010; Mőst et al., 2013)。近年来, 我国 学者利用分子手段研究发现, 长刺溞主要分布于东 部平原地区, 颈齿溞分布于西部乃至青藏高原( $\mathrm{Ma}$ et al., 2015), 并发现分布于西藏地区的盔型溞与平 原地区的类群间出现分化(徐磊, 2013)。因此, 青藏 高原的特殊生境以及历史气候条件使该地区的枝 角类多样性与内地平原地区有着明显的区别和分 化(蒋筊治等, 1983)。然而, Ma等(2015)的研究没有 在西藏地区发现长刺溞的分布, 因而缺少长刺溞在 西藏地区与东部平原地区的对比研究, 徐䂞(2013) 虽研究了盔形溞在西藏地区与平原地区的分化, 但 没有涉及复合种中的长刺溞和颈齿溞。本文以青藏 高原分布的长刺溞复合种为对象并结合其他地区 已经发表的序列信息, 利用分子系统学的方法, 研 究长刺溞复合种内的长刺溞、颈齿溞和盔型溞是否 与平原地区分布的种群存在分化, 以及不同种间的 分化差异是否相似, 并初步探讨其种群分化和地理 分布格局形成的原因。

\section{1 方法}

\section{1 样品采集与DNA提取}

2013年 8 月在西藏地区共调查了 42 个湖泊, 在 35 个湖中发现有枝角类, 但仅在班公错、沉错、错 鄂、拉萨河和打加芒错 5 个湖泊中发现了长刺溞复 合种(盔形溞、长刺溞、颈齿溞)。使用 $200 \mu \mathrm{m}$ 的浮 游动物网表层拖网进行样品采集, 用 $95 \%$ 乙醇现场 固定, $4^{\circ} \mathrm{C}$ 冰箱保存。

每个种群(对应每个湖泊)都在解剖镜下挑取 10 个以上个体。由于溞属动物外被甲壳, 在提取DNA 前先用灭菌的牙签捣碎溞体, 使用Ultra-Sep Gel Extraction Kit 试剂盒(OMEGA生物公司) 提取 DNA。具体的提取方法参考徐否等(2014), 提取的 
DNA置于 $-20^{\circ} \mathrm{C}$ 冰箱保存。

\section{$1.2 \mathrm{COI}$ 序列扩增与测序}

COI基因扩增引物为LCO1490 (5' GGT CAA CAA ATC ATA AAG ATA TTG G 3')和HCO2198 (5' TAA ACT TCA GGG TGA CCA AAA AAT CA 3') (Folmer et al., 1994)。扩增反应总体积为 $30 \mu \mathrm{L}$, 包 括: $3 \mu \mathrm{L} 10 \times$ buffer, $1.5 \mathrm{mM} \mathrm{MgCl}$, $0.2 \mathrm{mM}$ dNTPs, 引物各 $0.5 \mu \mathrm{M}, 0.5 \mathrm{U}$ Taq酶(TaKaRa)和 $3 \mu \mathrm{L}$ 模板 DNA。扩增条件为 $95^{\circ} \mathrm{C}$ 预变性 $1 \mathrm{~min} ; 95^{\circ} \mathrm{C}$ 变性 1 min, $94^{\circ} \mathrm{C}$ 变性 $40 \mathrm{~s}, 51^{\circ} \mathrm{C}$ 退火 $40 \mathrm{~s}, 72^{\circ} \mathrm{C}$ 延伸 $1 \mathrm{~min}$, 共 35 个循环; $72^{\circ} \mathrm{C} 5 \mathrm{~min}$ 充分延伸 $4^{\circ} \mathrm{C}$ 结束。扩增产 物经电泳检测后, 选择目的带清晰且明亮的PCR反 应产物送交华大基因(广州)纯化, 并测序。所有样品 均采用双向测序，正反向对比后确定分析用序列。

每个种群测序获得 10 条COI基因序列(每条COI 基因序列对应 1 个个体), 共 50 条序列, 去除每个种 群内部相同的序列后, 最终用于分析的个体(序列) 为 9 个(条)。分别是班公错 2 个, 错鄂 2 个, 打加芒错 2 个, 沉错 1 个, 拉萨河 2 个。

\section{3 数据处理和系统学分析}

\subsection{1 数据处理}

测序获得的COI基因序列先在 NCBI数据库中
进行BLAST搜索比对以保证序列的可靠性。另在 GenBank数据库中下载 9 条已发表的长刺溞复合种 的COI基因序列(表1), 有来自我国东部低海拔地区 的盔形溞、北美的颈齿溞和欧洲的长刺溞等序列。 用 BioEdit 7 软件及其附带的Clustal W比对软件将 实验获得的和GenBanK中下载的COI基因序列进行 同源比对, 并人工校对, 去除两端引物结合位点的 缺失序列, 获得的COI基因序列用于分析。

COI 基因序列差异情况特别是固化的差异 (fixed differences)在DnaSP 5.10软件(Rozas et al., 2003)中分析计算。序列的碱基组成、碱基替代以及 遗传差异度在MEGA 6.06 (Kumar et al., 2008)软件 中分析, 其中遗传差异度采用 Kimura 双参数 (Kimura 2-parameter双参模型)模型计算, 参数设置 如下: 变异估算方法为自举法(bootstrapping method), 运行次数为 1,000 , 核苷酸替代包括转换和颠 换, 序列间位点选择相同进化率, 删除缺失位点。 在HAPLOVIEWER中构建单倍型网络图(Salzburger et al., 2011)。

\subsection{2 系统学分析}

在进行系统学分析之前, 用Mrmodeltest 2.3软 件对经上述比对后的序列数据进行AIC最适核酸替

表1 长刺泽复合种种群位点及序列信息

Table 1 Geographic and genetic characteristics of 18 Daphnia longispina complex populations

\begin{tabular}{|c|c|c|c|c|c|}
\hline $\begin{array}{c}\text { 采样点 } \\
\text { Location }\end{array}$ & $\begin{array}{c}\text { 纬度 } \\
\text { Latitude }\end{array}$ & $\begin{array}{c}\text { 经度 } \\
\text { Longitude }\end{array}$ & $\begin{array}{l}\text { 代号 } \\
\text { Code }\end{array}$ & $\begin{array}{c}\text { GenBank登录号 } \\
\text { GenBank accession }\end{array}$ & $\begin{array}{c}\text { 序列来源 } \\
\text { Sequence source }\end{array}$ \\
\hline \multicolumn{6}{|l|}{ 盔形溞 Daphnia galeata } \\
\hline 错鄂 Cuoe, China & $31.63^{\circ} \mathrm{N}$ & $88.67^{\circ} \mathrm{E}$ & CE1 & & 本研究 This study \\
\hline 错鄂 Cuoe, China & $31.63^{\circ} \mathrm{N}$ & $88.67^{\circ} \mathrm{E}$ & CE2 & & 本研究 This study \\
\hline 打加芒错 Dajiamangcuo, China & $29.65^{\circ} \mathrm{N}$ & $85.75^{\circ} \mathrm{E}$ & DJMC1 & & 本研究 This study \\
\hline 打加芒错 Dajiamangcuo, China & $29.65^{\circ} \mathrm{N}$ & $85.75^{\circ} \mathrm{E}$ & DJMC2 & & 本研究 This study \\
\hline 骆马湖 Luoma Lake, China & $34.07^{\circ} \mathrm{N}$ & $118.11^{\circ} \mathrm{E}$ & HC3 & KM555356 & GenBank \\
\hline 西湖 West Lake, China & $30.15^{\circ} \mathrm{N}$ & $120.08^{\circ} \mathrm{E}$ & HC2 & KM555355 & GenBank \\
\hline 宝应湖 Baoying Lake, China & $33.10^{\circ} \mathrm{N}$ & $119.14^{\circ} \mathrm{E}$ & HC1 & KM555354 & GenBank \\
\hline \multicolumn{6}{|l|}{ 颈齿溞 Daphnia dentifera } \\
\hline 沉错 Chencuo, China & $28.88^{\circ} \mathrm{N}$ & $90.47^{\circ} \mathrm{E}$ & CC & & 本研究 This study \\
\hline 拉萨河 Lasahe, China & $29.68^{\circ} \mathrm{N}$ & $91.28^{\circ} \mathrm{E}$ & LS1 & & 本研究 This study \\
\hline 拉萨河 Lasahe, China & $29.68^{\circ} \mathrm{N}$ & $91.28^{\circ} \mathrm{E}$ & LS2 & & 本研究 This study \\
\hline 那曲湖 Naqu Lake, China & $31.48^{\circ} \mathrm{N}$ & $92.05^{\circ} \mathrm{E}$ & NQHA & KM555369 & GenBank \\
\hline Canada & & & CND & FJ427488 & GenBank \\
\hline 格桑桥 Gesangqiao, China & $29.65^{\circ} \mathrm{N}$ & $91.12^{\circ} \mathrm{E}$ & GSQA & KM555366 & GenBank \\
\hline \multicolumn{6}{|l|}{ 长刺溞 Daphnia longispina } \\
\hline 班公错 Bangongcuo, China & $33.71^{\circ} \mathrm{N}$ & $78.81^{\circ} \mathrm{E}$ & BGC1 & & 本研究 This study \\
\hline 班公错 Bangongcuo, China & $33.71^{\circ} \mathrm{N}$ & $78.81^{\circ} \mathrm{E}$ & BGC2 & & 本研究 This study \\
\hline Sweden & & & DEM & EF375861 & GenBank \\
\hline Germany & & & H29 & EF375860 & GenBank \\
\hline Switzerland & & & SZL & EF375862 & GenBank \\
\hline
\end{tabular}


换模型笁选(Posada \& Crandall, 2001; Irestedt et al., 2004), 得到最佳模型 $G T R+G$ 。贝叶斯系统进化树 的构建使用 MrBayes v.3.1.2 软件(Huelsenbeck \& Ronquist, 2001; Huelsenbeck et al., 2001)中马尔科 夫链的蒙特卡洛方法(MCMC) 完成, 参数设置如 下: 同时起始 4 条马尔科夫链, 即 3 条热链和 1 条 冷链, 以随机树为起始树, 共运行 2,000,000 次, 每 100 代抽样 1 次, 重复 1 次。在舍弃老化样本 (burnin sample)后, 根据剩余的样本构建一致树 (consensus tree)。以后验概率(posterior probability, $\mathrm{PP}$ )来表示各分支的可信度, $95 \%$ 以上时认为该支 可靠。最大似然法(maximum likelihood, ML)构建的 系统进化树是在 Paup* 4.0 beta 10 (Swofford, 2003) 软件中设定运行, 使用启发式搜索(heuristic search) 与 100 次重复的自举分析(100 bootstrap replicates) 检验各分支的置信度。两种方法构建的系统进化树 所选取的外群 (out-group)均为溞属的虫状溞 (D. pulex), 所有运算完成后的系统树均由 Treeview 1.6.6 (Page, 1996)展示并编辑。

\section{2 结果}

\section{1 序列组成分析}

从西藏地区获得的 9 条COI基因序列中(CE1、

CE2、DJMC1、DJMC2、BGC1、BGC2、LS1、

LS2、CC), 盔形溞分布于错鄂和打加芒错 2 个湖
泊，颈齿溞分布于拉萨河和沉错 2 个湖泊，而长刺 溞只在班公错中被发现(表1)。实验获得的与 GenBank下载的长刺溞复合种COI基因序列比对 结果显示, 其基因序列碱基 $\mathrm{A}+\mathrm{T}$ 含量(55.0\%)略 高于G + C含量(45.0\%), 序列有可识别位点(包括 缺失与丢失的位点) 617 个, 其中保守位点 488 个, 变异位点 129 个, 单一位点 9 个, 简约信息点 120 个。

\subsection{COI基因分歧度和单倍型}

颈齿溞种群内遗传差异度为 $0.33-2.32 \%$, 盔 型溞为 $0.33-2.74 \%$, 长刺溞最高, 为 $1.31-5.50 \%$ (表2-4)。盔形溞、长刺溞和颈齿溞的种间遗传差 异度为9.40-16.98\% (表5), 平均转换/颠换(si/sv) 数为3.06, 转换数明显高于颠换数。 3 个种的序列 固化差异结果显示: 颈齿溞(DD)组和盔形溞(DG) 组序列固化差异最大, 为79(80.61\%), 变异位点 为 107 个, 简约信息位点 98 个; 颈齿溞(DD)组和长 刺溞(DL)组最小, 为32(49.23\%), 变异位点为85 个, 简约信息位点为 65 个(表5)。

西藏地区获得的9条COI基因序列和GenBank 上下载的9条长刺溞复合种 $\mathrm{COI}$ 基因序列共产生15 个单倍型。单倍型网络图显示(图1), 长刺溞复合 种由 3 个明显的分支组成, 分别对应长刺溞、盔形 溞和颈齿溞。其中, 打加芒错种群盔形溞 2 条序列 共用同一个单倍型, 班公错长刺溞同一个种群中

表2 盔形溞(Daphnia galeata)种群间(对角线下)和种群内(对角线)的遗传差异度(\%)

Table 2 The genetic differentiation (\%) between (below the diagonal) and within (on the diagonal) Daphnia galeata populations

\begin{tabular}{lccccc}
\hline \multicolumn{7}{c}{ HC3 } & HC1 & HC2 & CE & DJMC \\
\hline HC3 & n.c. & & & & \\
HC1 & $0.33 \pm 0.23$ & n.c. & n.c. & & \\
HC2 & $2.15 \pm 0.57$ & $1.81 \pm 0.53$ & $0.90 \pm 0.36$ & $0.16 \pm 0.15$ & $0.16 \pm 0.15$ \\
CE & $2.74 \pm 0.66$ & $2.40 \pm 0.63$ & $0.65 \pm 0.33$ & $0.90 \pm 0.37$ & 0.15 \\
DJMC & $2.49 \pm 0.62$ & $2.15 \pm 0.59$ & & & \\
\hline
\end{tabular}

种群代号同表1。

The population codes are the same as those in Table 1 .

表3 长刺溞(Daphnia longispina)种群间(对角线下)和种群内(对角线)的遗传差异度(\%)

Table 3 The genetic differentiation (\%) between (below the diagonal) and within (on the diagonal) Daphnia longispina populations

\begin{tabular}{lcccc}
\hline & DEM & H29 & SZL & BGC \\
\hline DEM & n.c. & & & \\
H29 & $1.81 \pm 0.53$ & n.c. & n.c. & $1.62 \pm 0.51$ \\
SZL & $1.81 \pm 0.54$ & $1.31 \pm 0.43$ & $4.62 \pm 0.80$ & \\
BGC & $5.50 \pm 0.92$ & $4.88 \pm 0.84$ & \\
\hline
\end{tabular}

种群代号同表1。

The population codes are the same as those in Table 1 . 
表4 颈齿溞(Daphnia dentifera)种群间(对角线下)和种群内(对角线)的遗传差异度(\%)

Table 4 The genetic differentiation (\%) between (below the diagonal) and within (on the diagonal) Daphnia dentifera populations

\begin{tabular}{lccccc}
\hline \multicolumn{7}{c}{ CND } & NQHA & GSQA & LS & CC \\
\hline CND & n.c. & & & & \\
NQHA & $1.98 \pm 0.55$ & n.c. & & & \\
GSQA & $2.15 \pm 0.60$ & $0.49 \pm 0.30$ & n.c. & & \\
LS & $2.32 \pm 0.60$ & $0.65 \pm 0.30$ & $0.33 \pm 0.16$ & $0.65 \pm 0.32$ & n.c. \\
CC & $2.15 \pm 0.60$ & $0.49 \pm 0.30$ & 0.00 & $0.33 \pm 0.16$ & \\
\hline
\end{tabular}

种群代号同表1。

The population codes are the same as those in Table 1.

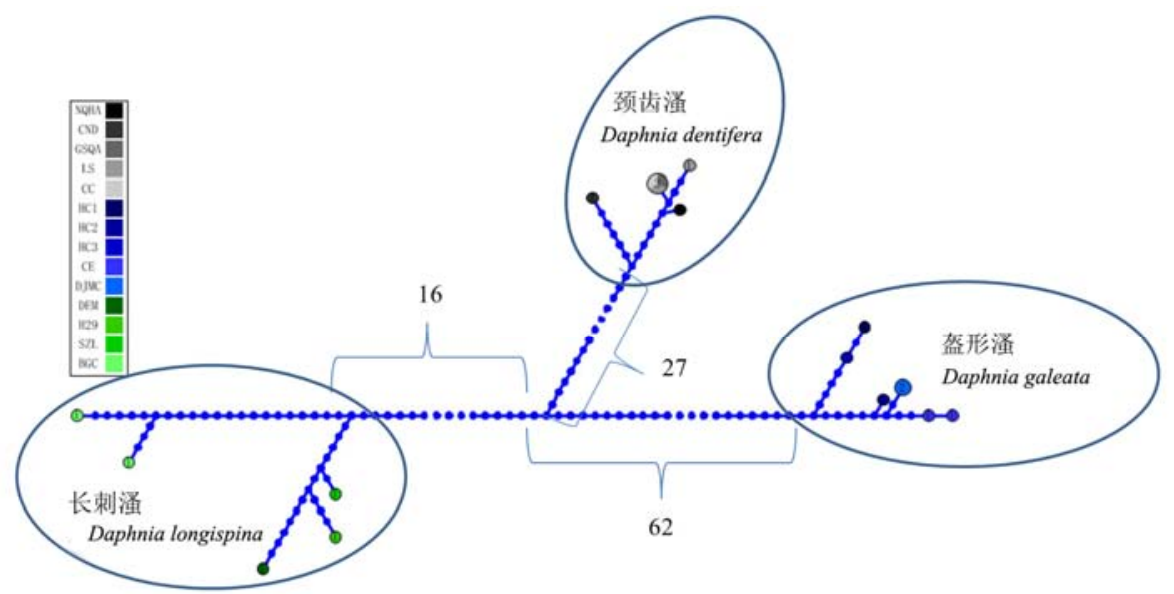

图1 基于COI基因序列构建的长刺泽复合种单倍型网络图(图中数字代表了各分支的平均突变数量)。图例中的缩写参见表1。 Fig. 1 Haplotype network for Daphnia longispina complex based on mitochondria COI gene sequences. Numbers between subnetworks represent the average number of mutations between subclades. The population codes are the same as those in Table 1.

表5 长刺泽复合种(Daphnia longispina complex)种间和种 内的遗传差异度 $(\%)$ (对角线和对角线下)及复合种间各单倍 型的固化差异(对角线上)

Table 5 The genetic differentiation (\%) between and within species in Daphnia longispina complex (on the diagonal and below the diagonal) and the fixed differences among these haplotypes (above the diagonal)

\begin{tabular}{cccc}
\hline & DG & DL & DD \\
\hline DG & $1.46 \pm 0.31$ & $71(67.62 \%)$ & $79(80.61 \%)$ \\
DL & $16.70 \pm 1.72$ & $3.66 \pm 0.59$ & $32(49.23 \%)$ \\
DD & $16.98 \pm 1.87$ & $9.40 \pm 1.12$ & $1.01 \pm 0.24$ \\
\hline
\end{tabular}

代号为拉丁种名的缩写。 DG是D. galeata, DL是 D. longispina, DD 是D. Dentifera。

The code is the abbreviation of the species Latin name. DG is $D$. galeata, DL is D. longispina, and DD is D. Dentifera.

有2个不同的单倍型, 且2个单倍型序列种内差异与 欧洲地区单倍型序列差异较国内盔形溞和颈齿溞 序列大。

\section{3 系统学分析}

最大似然法构建的系统进化树与贝叶斯系统
进化树的分支基本一致，用一根进化树表示(图2), 都是由 3 个明显进化支组成, 分别对应长刺溞、盔型 溞及颈齿溞。3 3 个大的分支的贝叶斯算法得到的后 验概率高于 0.9 , 最大似然法得到的自举值也都 $\geqslant$ $70 \%$, 说明各分支出现的可靠性较高。

\section{3 讨论}

\section{1 长刺溞复合种内部分化}

长刺溞复合种是溞属分布最为广泛的种类之 一, 分布于欧亚大陆以及北美大陆, 其形态可塑性 极强, 体型和头盔变化较大, 分类特征不明显 (Benzie, 2005), 传统的形态分类学方法难以发现隐 种的存在。线粒体DNA作为严格的母性遗传, 具有 变异快、选择压力小的特征, 通过探查其序列变化 可有效发现 2 个在形态学上难以区分的种类在DNA 水平上的差异, 并通过序列差异度, 建立物种在 不同分类单元的分类标准, 即DNA条形码(DNA 


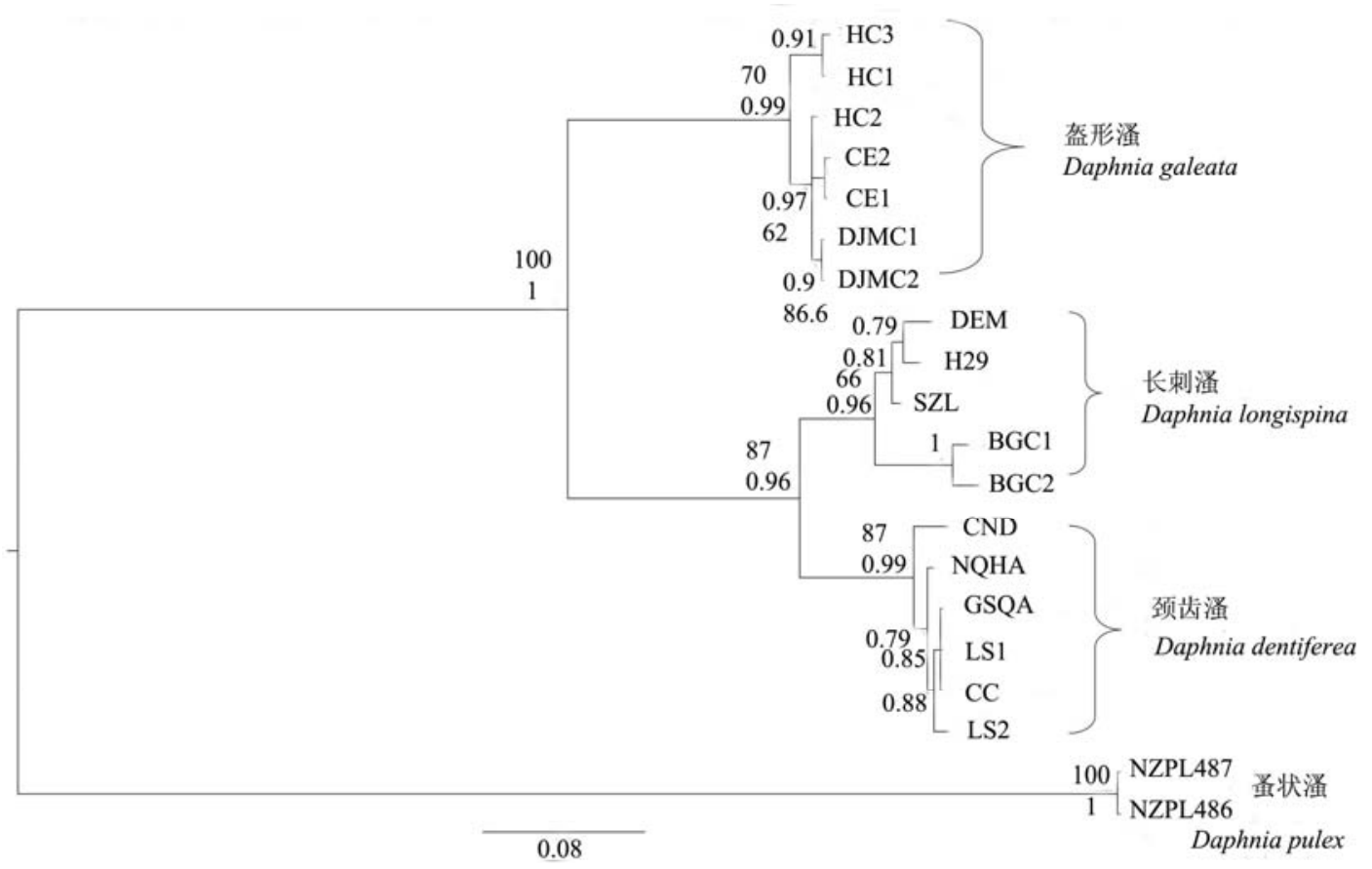

图2 基于COI基因序列构建长刺掻复合种贝叶斯系统树与最大似然法系统树组合的进化树, 贝叶斯系统树后验概率(PP)和 最大似然法系统进化树的自举支持率(bootstrap values)(百分数)均标于树分支的上面。图中的缩写为表1中COI序列代号。

Fig. 2 Bayes and Maximum Likelihood phylogenetic tree of Daphnia longispina complex based on mitochondria COI gene sequences. Posterior probabilities (Bayes tree) and bootstrap values (Maximum Likelihood tree) are shown above the nodes. The population codes are the same as those in Table 1.

barcodes)(Hebert et al., 2003a)。COI 基因是使用最 广泛的DNA条码, 得益于GenBank和BOLD强大的 数据量, 不同种类的生物可以通过比较COI基因差 异度来确定分化水平(K2P双参模型)。甲壳纲的属 内分化平均差异度为 $17.16 \%$, 枝角类中溞属的属内 种间平均差异度最大, 变化范围在13.18-30.56\%, 平均高达 $25.28 \%$, 溞属种内最高为 $4.3 \%$, 平均 1.32\% (Costa et al., 2007)。本研究通过COI基因构建 的系统树显示, 长刺溞复合种由 3 个明显进化支组 成, 分别对应长刺溞、盔型溞和颈齿溞(图2), 三者 之间的遗传差异度(K2P双参模型)为9.40-16.98\% (表5), 远远高于甲壳类种内平均差异度 $(0.46 \%)$, 也 高于溞属种内最高遗传差异度(4.3\%), 却未达到溞 属种类种间分化平均水平 $(25.28 \%)$, 其中长刺溞与 颈齿溞的遗传距离仅为 $9.4 \%$, 甚至低于溞属种类种 间分化最低水平(13.18\%) (Costa et al., 2007; Adamowicz et al., 2009), 说明这3个溞属种类的分化介 于种与属的分化之间, 故以复合种的形式存在。

\section{2 西藏地区长刺泽复合种分化}

本研究显示, 长刺溞复合种中分布最广的 3 个
种，即盔型溞、长刺溞和颈齿溞，在西藏地区均有 分布。西藏地区湖泊面积超过全国湖泊总面积的 $1 / 4$, 不仅是中国湖泊面积最大、最为集中的地区之 一, 也是世界上海拔最高、数量最多和面积最大的 内陆高原湖区(张继承等, 2008)。本次调查了 40 多个 湖泊，但仅在班公错、沉错、拉萨河、错鄂和打加 芒错 5 个湖泊中发现了长刺溞复合种。三个种的平 均差异度都高于溞属内的平均水平 $1.32 \%$, 说明盔 型溞和颈齿溞虽然没有达到种间的分化，但已呈现 出亚种分化的趋势。类似的种内分化在溞属的其他 种类中也有发现，北美D. ambigua和D. laevis种内 不同地理分布类群的线粒体DNA的分歧度也都超 过2\%, 地理隔离是导致分化的主要原因(Taylor et al., 1998; Hebert et al., 2003b)。

值得注意的是, 长刺溞的分化已超过溞属种内 最高遗传差异度(4.3\%), 三个种遗传差异度的不同 可能与其历史扩散和分布格局有关。第四纪冰期, 随着冰川活动的加剧, 北方及高海拔地区被冰雪覆 盖，使得分布于欧洲的长刺溞南迁至地中海沿岸的 利比利亚半岛、亚平宁半岛和巴尔干半岛, 这三个 
地区也即欧洲著名的冰期生物避难所(Sommer \& Zachos, 2009), 因此与东亚地区的长刺溞失去交流 机会。然而, 第四纪冰川活动也使得海平面下降, 阿 拉斯加与西伯利亚通过陆桥相连, 使得古北区的盔 型溞有机会在更新世可以扩散至新北区, 并与北美 分布的颈齿溞种发生基因融合(Ishida \& Taylor, 2007), 随着更新世冰期间冰期的反复循环, 分布于 不同生物避难所的长刺溞复合种分布范围会有所变 化, 但长达几万年甚至十几万年的冰期时间, 使得 不同区系间的盔型溞失去基因交流的机会, 产生了 异域分化，最终形成了目前的系统地理格局。

\section{3 我国长刺泽复合种系统地理学分布格局}

早期长刺溞复合种的地理分布研究都基于野 外调查和形态鉴定, 但是长刺溞复合种内的几个种 形态学鉴定较为困难(Kotov, 2015), 三者从形态上 难以区分, 容易混淆; 其中颈齿掻的鉴定更需要雄 体的特征(雄体颈后部有齿状结构) (Benzie, 2005), 而雄体样本通常不易获得, 国外学者也曾经把颈齿 溞归为 $D . r o s e a$ 。直到1996年, Taylor等(1996)通过 $12 \mathrm{~S}$ rDNA和等位酶对长刺溞复合种进行研究, 才 将颈齿溞从北美的D. rosea中划分出来。我国早期的 记录显示长刺溞分布较广, 在我国大部分省份和地 区都有分布(蒋卒治和堵南山, 1979); 由于鉴定较 困难, 以及早期的调查研究缺乏分子生物学辅助信 息, 因此有理由怀疑过去我国记录显示的长刺溞分 布范围, 实际上也包括了盔型溞或者颈齿溞, 因此 并未将二者单独加以区分和详细描述。而近期分子 系统地理学的研究显示(徐磊, 2013; Ma et al., 2015), 盔型溞与颈齿溞在我国的分布更广, 在 $\mathrm{Ma}$ 等(2015)对中国分布的长刺溞复合种的研究中, 18 个种群中甚至没有发现长刺溞的分布。

在本研究中长刺溞只在西藏班公错有发现，其 余样本皆为盔型溞和颈齿溞。从分布上看, 盔型溞 主要分布于东部低纬度的平原地区, 而颈齿溞分布 于西部高海拔的青藏高原地区, 这与Ishida等(2011) 的研究结果类似, 盔型溞主要分布于低纬度或低海 拔地区, 颈齿溞主要分布于高纬度或高海拔地区, 说明温度是影响长刺溞复合种分布的主要原因之 一。盔型泽更倾向于分布在温度较高的水体中, 而 颈齿溞主要分布于温度较低的高山湖泊(Spaak et al., 2012)。除此之外, 鱼类的捕食压力和水体营养 水平也是影响长刺溞复合种分布格局的原因, 盔型
溞头盔的形态变化以及垂直迁移能力使其可以大 量存在于中富营养以及鱼类捕食压力较强的水体 (Dodson, 1990; Sekino \& Yoshioka, 1995), 本研究中 错鄂为内陆中营养水平的淡水湖泊, 湖内水生植物 生长茂盛, 鱼类、鸟类资源丰富。颈齿掻仅存在于 西藏地区寡营养水体中(Ma et al., 2015), 沉错处于 喜马拉雅山北坡的山间盆地, 湖面海拔为 $4,438 \mathrm{~m}$, 湖水主要由卡鲁雄曲河引来的冰川融水补给, 湖水 常年温度较低。由此可见, 盔型溞和颈齿溞在我国 西藏地区的分布与欧洲高山湖泊的研究结果相似, 水体温度和捕食压力可能是影响二者分布的主要 原因。而对长刺溞而言, 由于样本较少, 对于其分 布格局的研究需要进一步的数据支持。

\section{参考文献}

Adamowicz SJ, Purvis A (2005) How many branchiopod crustacean species are there? Quantifying the components of underestimation. Global Ecology and Biogeography, 14, 455-468.

Adamowicz SJ, Petrusek A, Colbourne JK, Hebert PDN, Witt JDS (2009) The scale of divergence: a phylogenetic appraisal of intercontinental allopatric speciation in a passively dispersed freshwater zooplankton genus. Molecular Phylogenetics and Evolution, 50, 423-436.

Allen MR, Thum RA, Caceres CE (2010) Does local adaptation to resources explain genetic differentiation among Daphnia populations? Molecular Ecology, 19, 3076-3087.

Benzie JAH (1988) The systematics of Australian Daphnia (Cladocera: Daphniidae). Electrophoretic analyses of the Daphnia carinata complex. Hydrobiologia, 166, 183-197.

Benzie JAH (2005) Cladocera: the genus Daphnia (including Daphniopsis) (Anomopoda: Daphniidae). Guides to the identification of the microinvertebrates of the continental waters of the world. Quarterly Review of Biology, 80, 491-510.

Chiang SC (蒋䁨治), Du NS (堵南山) (1979) Fauna Sinica, Crustacea, Freshwater Cladocera (中国动物志 - 淡水枝角 类). Science Press, Beijing. (in Chinese)

Chiang SC (蒋䁨治), Shen YF (沈暳芬), Gong XJ (龚循矩) (1983) Aquatic Invertebrates of the Tibetan Plateau (西藏水 生无脊椎动物). Science Press, Beijing. (in Chinese)

Colbourne JK, Crease TJ, Weider LJ, Hebert PDN, Dufresne F, Hobaek A (1998) Phylogenetics and evolution of a circumarctic species complex (Cladocera: Daphnia pulex). Biological Journal of the Linnean Society, 65, 347-365.

Costa FO, Jeremy RD, James B, Rantnasingham S, Dooh RT, Hajibaei M, Hebert PDN (2007) Biological identifications through DNA barcodes: the case of the Crustacea. Canadian Journal of Fisheries and Aquatic Sciences, 64, 272-290.

De Meester L (1996) Local genetic differentiation and adapta- 
tion in freshwater zooplankton populations: patterns and processes. Ecoscience, 3, 385-399.

Dlouhá S, Thielsch A, Kraus RHS, Seda J, Schwenk K, Petrusek A (2010) Identifying hybridizing taxa within the Daphnia longispina species complex: which methods to rely on? Hydrobiologia, 643, 107-122.

Dodson S (1990) Predicting diel vertical migration of zooplankton. Limnology and Oceanography, 35, 1195-1200.

Dumont HJ, Negrea S (2002) Introduction to the Class Brachiopoda, Guides to the Identification of the Microinvertebrates of the Continental Waters of World 19. Backhuys Publishers, Leiden.

Folmer O, Black M, Hoeh W, Lutz R, Vrijenhoek R (1994) DNA primers for amplification of mitochondrial cytochrome c oxidase subunit I from diverse metazoan invertebrates. Molecular Marine Biology and Biotechnology, 3, 294-299.

Forro' L, Korovchinsky NM, Kotov AA, Petrusek A (2008) Global diversity of cladocerans (Cladocera; Crustacea) in freshwater. Hydrobiologia, 595, 177-184.

Giessler S (1997a) Analysis of reticulate relationships within the Daphnia longispina species complex. Allozyme phenotype and morphology. Journal of Evolutionary Biology, 10, 87-105.

Giessler S (1997b) Gene flow in the Daphnia longispina hybrid complex (Crustacea, Cladocera) inhabiting large lakes. $\mathrm{He}$ redity, $\mathbf{7 9}, 231-241$.

Hebert PHD (1978) The population biology of Daphnia (Crustacea, Daphnidae). Biological Reviews, 53, 387-426.

Hebert PDN, Cywinska A, Ball SL, De Waard JR (2003a) Biological identifications through DNA barcodes. Proceedings of the Royal Society B: Biological Sciences, 270, 313-321.

Hebert PDN, Witt JDS, Adamowicz SJ (2003b) Phylogeographical patterning in Daphnia ambigua: regional divergence and intercontinental cohesion. Limnology and Oceanography, 48, 261-268.

Huelsenbeck JP, Ronquist F (2001) MRBAYES: Bayesian inference of phylogeny. Bioinformatics, 17, 754-755.

Huelsenbeck JP, Ronquist F, Nielsen R, Bollback JP (2001) Bayesian inference of phylogeny and its impact on evolutionary biology. Science, 294, 2310-2314.

Hutchison GE (1967) A Treatise on Limnology. PhD dissertation, National Academy of Sciences, New York.

Irestedt M, Fjeldså J, Nylander J, Ericson P (2004) Phylogenetic relationships of typical antbirds (Thamnophilidae) and test of incongruence based on Bayes factors. BMC Evolutionary Biology, 4, 1-16.

Ishida S, Taylor DJ (2007) Quaternary diversification in a sexual Holarctic zooplankter, Daphnia galeata. Molecular Ecology, 16, 569-582.

Ishida S, Takahashi A, Matsushima N, Yokoyama J, Makino W, Urabe J, Kawata M (2011) The long-term consequences of hybridization between the two Daphnia species, $D$. galeata and D. dentifera, in mature habitats. BMC Evolutionary Biology, 11, 209-222.

Korovchinsky NM (1996) How many species of Cladocera are there? Hydrobiologia, 321, 191-204.
Kotov AA (2015) A critical review of the current taxonomy of the genus Daphnia O. F. Müller, 1785 (Anomopoda, Cladocera). Zootaxa, 3911, 184-200.

Kotov AA, Ishida S, Taylor DJ (2006) A new species in the Daphnia curvirostris (Crustacea: Cladocera) complex from the eastern Palearctic with molecular phylogenetic evidence for the independent origin of neckteeth. Journal of Plankton Research, 28, 1067-1079.

Kumar S, Dudley J, Nei M, Tamura K (2008) MEGA: a biologist-centric software for evolutionary analysis of DNA and protein sequence. Briefings in Bioinformatics, 9, 299-306.

Lampert W (2011) Daphnia: Development of Model Organism in Ecology and Evolution. American Institute of Biological Sciences, Washington DC.

Ma XL, Petrusek A, Wolinska J, Gießler S, Zhong Y, Hu W, Yin MB (2015) Diversity of the Daphnia longispina species complex in Chinese lakes: a DNA taxonomy approach. Journal of Plankton Research, 37, 56-65.

Mergeay J, Aguilera X, Declerck S, Petrusek A, Huyse T, De Meester L (2008) The genetic legacy of polyploid Bolivian Daphnia: the tropical Andes as a source for the North and South American Daphnia pulicaria complex. Molecular Ecology, 17, 1789-1800.

Mőst M, Petrusek A, Sommaruga R, Juračka PJ, Slusarczyk M, Manca M (2013) At the edge and on the top: molecular identification and ecology of Daphnia dentifera and $D$. longispina in high-altitude Asian lakes. Hydrobiologia, 715, 169-180.

Page RDM (1996) TREEVIEW: an application to display phylogenetic trees on personal computers. Computer Applications in the Biosciences, 12, 357-358.

Petrusek A, Cerny M, Mergeay J, Schwenk K (2007) Daphnia in the Tatra Mountain lakes: multiple colonization and hidden species diversity revealed by molecular markers. Fundamental and Applied Limnology, 169, 279-291.

Petrusek A, Hobæk A, Nilssen JP, Skage M, Černý M, Brede N, Schwenk K (2008) A taxonomic reappraisal of the European Daphnia longispina complex (Crustacea, Cladocera, Anomopoda). Zoologica Scripta, 37, 507-519.

Petrusek A (2007) Diversity of European Daphnia on Different Scales: from Cryptic Species to Within-lake Differentiation. $\mathrm{PhD}$ dissertation, Charles University, Prague.

Posada D, Crandall KA (2001) Selecting the best-fit model of nucleotide substitution. Systematic Biology, 50, 580-601.

Rozas J, Sanchez-Delbarria JC, Messeguer X (2003) DnaSP, DNA polymorphism analyses by the coalescent and other methods. Bioinformatics, 19, 2496-2497.

Salzburger W, Ewing GB, Haeseler VA (2011) The performance of phylogenetic algorithms in estimating haplotype genealogies with migration. Molecular Ecology, 20, 19521963.

Sekino T, Yoshioka T (1995) The relationship between nutritional condition and diel vertical migration of Daphnia galeata. Japanese Journal of Limnology, 56, 145-150.

Skage M, Hobæk A, Ruthová Š, Keller B, Petrusek A, Sed’a J, Spaak P (2007) Intra-specific rDNA-ITS restriction site var- 
iation and an improved protocol to distinguish species and hybrids in the Daphnia longispina complex. Hydrobiologia, 594, 19-32.

Sommer RS, Zachos FE (2009) Fossil evidence and phylogeography of temperate species: glacial refugia and post-glacial recolonization. Journal of Biogeography, 36, 2013-2020.

Spaak P, Fox J, Hairston NG Jr (2012) Modes and mechanisms of a Daphnia invasion. Proceedings of the Royal Society B: Biological Sciences, 279, 2936-2944.

Swofford D (2003) PAUP* 4.0 [Electronic Resource]: Phylogenetic Analysis Using Parsimony. Sinauer Associates Publishers, Sunderland.

Taylor DJ, Hebert PD, Colbourne JK (1996) Phylogenetics and evolution of the Daphnia longispina group (Crustacea) based on 12S rDNA sequence and allozyme variation. Molecular Phylogenetics and Evolution, 5, 495-510.

Taylor DJ, Finston TL, Hebert PDN (1998) Biogeography of a widespread freshwater crustacean: pseudocongruence and cryptic endemism in the North American Daphnia laevis complex. Evolution, 52, 1648-1670.

Ventura M, Petrusek A, Miró A, Hamrová E, Buñay D, De Meester L, Mergeay J (2014) Local and regional founder effects in lake zooplankton persist after thousands of years despite high dispersal potential. Molecular Ecology, 23, 1014-1027.

Xu L (徐否), Li SJ (李思嘉), Wang S (王晟), Han XY (韩小 玉), Han BP (韩博平) (2014) Comparison of two methods for extracting DNA of resting eggs by Cladocera. Journal of Lake Sciences (湖泊科学), 26, 632-636. (in Chinese with English abstract)

Xu L (徐否) (2013) Biogeography and Genetic Diversity of Two Cladoceran Species (Leptodora kindtii and Daphnia galeata) (两种枝角类 (Leptodora kindtii 和 Daphnia galeata) 的生物地理学及其种群遗传多样性研究). $\mathrm{PhD}$ dissertation, Jinan University, Guangzhou. (in Chinese with English abstract)

Zhang JC (张继承), Jiang QG (姜琦刚), Li YH (李远华), Wang K (王坤) (2008) Dynamic monitoring and climatic background of lake changes in Tibet based on RS/GIS. Journal of Earth Sciences and Environment (地球科学与环 境学报), 30, 285-292. (in Chinese with English abstract)

(责任编委：张德兴 责任编辑：问文杰) 